\title{
Modeling Transcranial DC Stimulation
}

\author{
Thom F. Oostendorp, Yvonne A. Hengeveld, Carsten H. Wolters, Jeroen Stinstra, \\ Gijs van Elswijk, Dick F. Stegeman
}

\begin{abstract}
A$ method to estimate the potential and current density distribution during transcranial DC stimulation (tDCS) is introduced. The volume conductor model consists of a realistic head model (concerning shape as well as conductivity), obtained from T1-, PD- and DT-MR images. The model includes five compartments with different conductivities. For the skull and the white matter compartments, the conductivities are anisotropic. Using this model, the potentials inside the head that are generated by tDCS electrodes positioned on the scalp were computed by using the Finite Element Method. The results show that this is a promising method for the study of the effects of tDCS.
\end{abstract}

\section{INTRODUCTION}

In transcranial direct current stimulation (tDCS), a weak direct current (usually $\leq 1 \mathrm{~mA}$ ) is applied to the human cortex by means of a pair of relatively large electrode pads (typically $5 \times 7 \mathrm{~cm}$ ) positioned on the scalp. By means of tDCS, changes in cortical excitability can be induced. For motor function, these changes can persist for up to one hour after the end of stimulation, provided that the stimulation has been applied sufficiently long (in the order of $10 \mathrm{~min}$ ) [1], [2].

The polarity of the stimulating current determines whether the excitability is increased or decreased. If the anode is placed over the cortical region of interest (anodal tDCS), the excitability is increased, whereas cathodal tDCS decreases the excitability. These modulations have mainly been studied by stimulating the primary motor cortex, for which the effects can be assessed by observing the responses to transcranial magnetic stimulation (TMS) before and after tDCS. The precise mechanism underlying the long-term effects are not clear, although some suggestions can be found in the literature [1]-[3].

Because tDCS is safe [4], cheap and easy to apply, it has been suggested as a method for inducing neuroplastic

This work was made possible in part by software from the NIH/NCRR Center for Integrative Biomedical Computing, P41-RR12553-07."

T. Oostendorp is with the Dept. Cognitive Neuroscience, Radboud University Medical Center in Nijmegen, the Netherlands (e-mail: t.oostendorp@science.ru.nl).

Y. Hengeveld, G. van Elswijk and D. Stegeman are with the Dept. of Clinical Neurophysiology, Radboud University Medical Center in Nijmegen, the Netherlands.

C. Wolters is with the Institute for Biomagnetism and Biosignalanalysis, at the University of Münster, Germany.

J. Stinstra is with the Scientific Computing and Imaging Institute, at the University of Utah, USA. changes of cortical functions. Reduction of symptoms in depression, chronic pain syndromes, stroke, epilepsy and Parkinson's disease have been reported (for a recent review, see Fregni and Pascual-Leone [5]).

It is obvious that it will not be easy to stimulate only a small region within the cortex by tDCS, nor will it be easy to determine the exact position and extent of the region that is affected. Some effects of the size of the stimulation electrodes have been observed in in vivo experiments [6]: reducing the size of the stimulation electrode at the region of interest, while keeping the applied current constant, results in increased focality of tDCS. Increasing the size of the reference electrode has the same effect.

Clearly, there is a need for modeling the distribution of the potential and the electric current density within the head for different electrode configurations. Some modeling studies have already been performed, but they did not take essential properties into account, like the anisotropy of parts of the volume conductor [7], [8], the shape of the volume conductor by using a spherical head model [9], or by modeling point electrodes instead of pads [8].

In the field of solving the forward and inverse problem in EEG and MEG there is a wide experience in using accurate head models [10], [11]. This paper describes a pilot study to

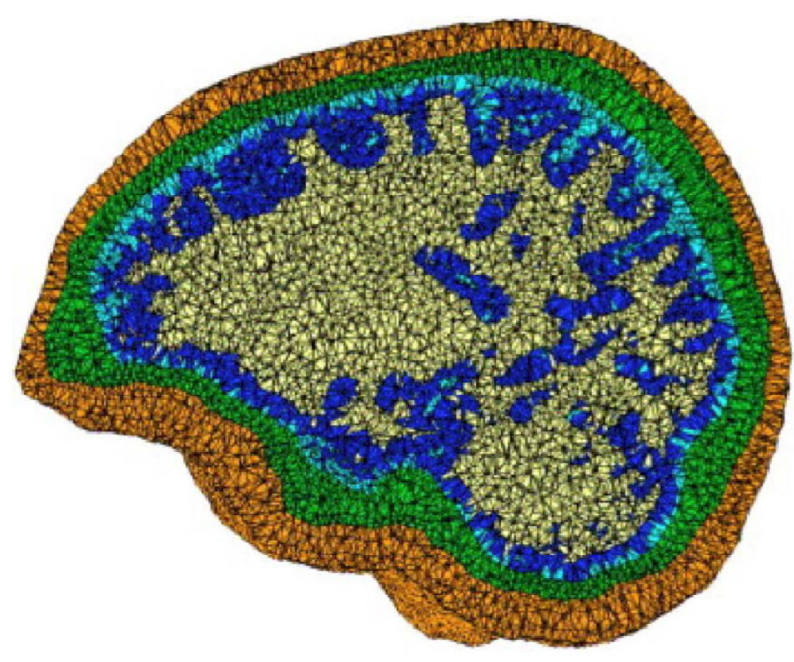

Fig. 1. Saggital cross-section of the head model. The different colors refer to different tissue types. At the top and the back of the head, the boundary of the model follows the true boundary of the head. In the facial and bottom part, the model consists of a layer of skin surrounding the skull (i.c.: the facial part is omitted from the model). (Reprinted from [11], Copyright (2006), with permission from Elsevier) 
TABLE 1

CONDUCTIVITY VALUES

\begin{tabular}{lll}
\hline \hline Tissue type & $\begin{array}{c}\text { Conductivities isotropic } \\
\text { model }(\mathrm{S} / \mathrm{m})\end{array}$ & $\begin{array}{c}\text { Conductivities anistropic } \\
\text { model }(\mathrm{S} / \mathrm{m})\end{array}$ \\
\hline Skin & 0.33 & 0.33 \\
Skull & 0.102 & 0.022 (radial) \\
& & 0.22 (tangential) \\
CSF & 1.79 & 1.79 \\
Gray matter & 0.33 & 0.33 \\
White matter & 0.14 & 0.065 (transverse) \\
& & 0.65 (longitudinal) \\
\hline \hline
\end{tabular}

Table I. Conductivity values of the different tissues. For the skull and the white matter both isotropic and anisotropic (ratios 1/10) conductivities were evaluated. Isotropic conductivity of the skull was reconstructed from the radial value of $1 / 15$ of the skin and gray matter conductivity [12], using a volume constraint as described by Wolters et al. [11]

modeling tDCS that applies a volume conductor model and a computational method as used in that field.

\section{METHODS}

\section{A. Volume conductor model}

The volume conductor model used in this study was developed by Wolters et al. in order to study the forward and inverse problem of EEG by finite element modeling [11]. MR images of the head of a 32-year old male subject were recorded. The voxels of the recorded data set were segmented into five distinctive tissues: scalp, skull, cerebrospinal fluid (CSF), and gray and white brain matter. The inner skull boundary was obtained from Proton Density (PD) $\mathrm{MR}$, while the other tissue boundaries were obtained from T1-weighted MR. The segmentation process is described in more detail in [11].

The software package CURRY was used to obtain a tetrahedral tessellation of the head in which all elements correspond to exactly one tissue type. The resulting mesh consisted of 117,287 vertices and 892,115 tetrahedra (fig. 1). The conductivities that were assigned to the tissues are listed in table I. The conductivity values were taken from the literature [12]-[14]. For the relation between the isotropic and the anisotropic conductivity tensor eigenvalues, a volume constraint was used [11]. For the skull, the conductivity in the direction perpendicular to the skull (i.e. radial direction) was assumed to be smaller than in the direction along the skull. For the white matter, the conductivity perpendicular to the fiber direction was assumed to be smaller than along the fiber direction. The fiber direction was estimaled from the diffusion tensors of water, as determined from Diffusion Tensor Imaging (DTI) using a U-flare protocol as described in Wolters et al. [11].

\section{B. Forward computations}

As the volume conductor model incorporates anisotropic conductivities, the often used Boundary Element Method (BEM) cannot be used. Instead, we applied the Finite Element Method (FEM) [15].

We have used the implementation of the FEM in the SCIRun package, that is freely available from the web under the MIT license (http://software.sci.utah.edu). This implementation treats bioelectric problems using the quasistatic approach, which applies well to DC stimulation.

In the case of tDCS there are no internal sources in the volume conductor, so the potential $\varphi$ inside the volume conductor obeys Laplace's equation:

$$
\vec{\nabla} \cdot(\sigma \vec{\nabla} \varphi)=0
$$

where $\sigma$ is the conductivity tensor.

The forward problem for tDCS is a mixed houndary value problem. At the electrode locations the potentials are impressed and the current density through the boundary is to be determined (Dirichlet boundary condition), whereas at the rest of the boundary, the potential is to be determined and the current densily through the boundary is zero (Neumann boundary condition):

$$
(\sigma \vec{\nabla} \varphi) \cdot \hat{n}=0
$$

where $\hat{n}$ is the normal at the surface.

The electrode locations in the model were determined by projecting two $5 \times 9 \mathrm{~cm}$ rectangles (electrode dimensions as provided by the company) onto the head model at the proper locations and orientations (see section C). (So, elements that are located at one of the projected electrodes are considered to obey Dirichlet boundary conditions with the potential set to the potential of the corresponding electrode, and all other elements at the surface are considered to obey Neumann

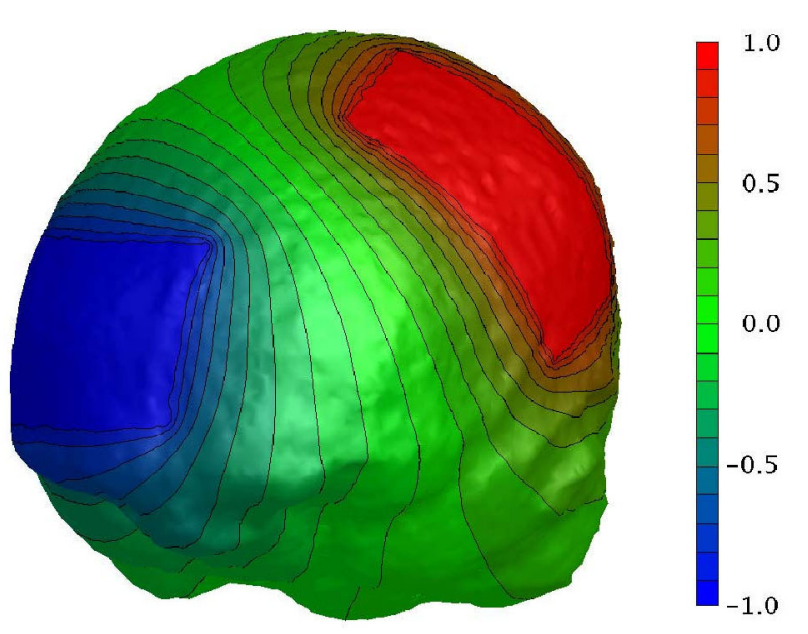

Fig. 2. Potential distribution on the scalp during stimulation (anisotropic model). Inner contours coincide with the electrodes used as cathode (blue) and as anode (red). 


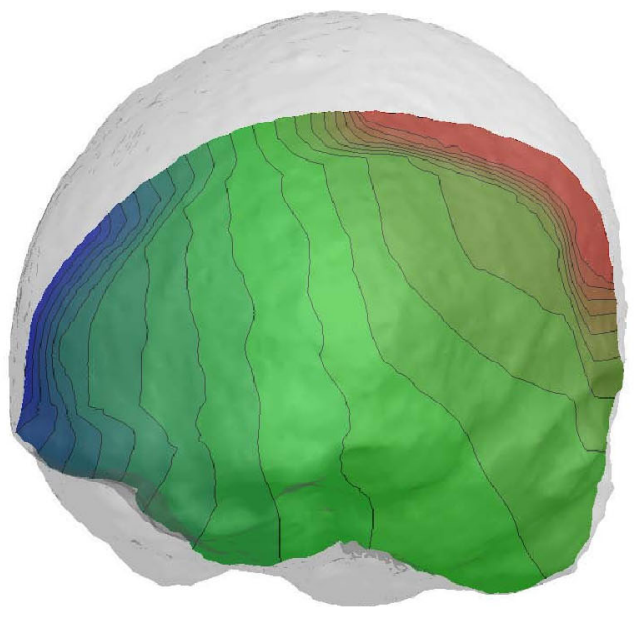

$\mathbf{A}$

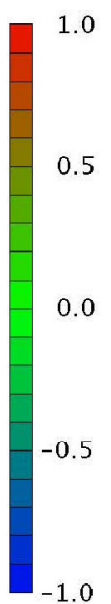

.0

Fig. 3. Potential distribution within the head at a plane that intersects both electrodes, for A: the anisotropic model and B: the isotropic model.

\section{boundary conditions.)}

SCIRun was used to compute the potential at all vertices for these boundary conditions. The current density within the head was determined by first computing the gradient of the potential at all elements, and multiplying the result with the conductivity tensors.

\section{Experiment}

For a number of healthy subjects tDCS (Eldith, Ilmenau, Germany) was performed. The active electrode was positioned above the left motor cortex, and the reference electrode above the right brow. During DC stimulation, the potential at the scalp was recorded using an EEG electrode configuration. A correct prediction of the potential distribution over the head is a first necessary test on the validity of the model outcome. The potentials generated by the DC stimulation are several orders of magnitude larger than the EEG, so in this way a recording of the potential distribution at the scalp generated by the tDCS is obtained.

\section{RESUlts}

Fig. 2 shows the computed potential distribution at the scalp during IC stimulation. 'The potential of the electrodes was set to -1 and 1 respectively (arbitrary units). Obviously, the potential varies gradually between the values at the stimulation electrodes. The performed EEG measurements correspond well to the presented potential profile. The precise way in which the potential varies along the surface depends on the values of the conductivities. However, the sensitivity of the head surface potential distribution appears to be relatively small. This, and a detailed comparison for validation of the FEM model will be studied in more detail in the follow-up of this research.

Fig. 3 shows the potential in a cross section of the head for

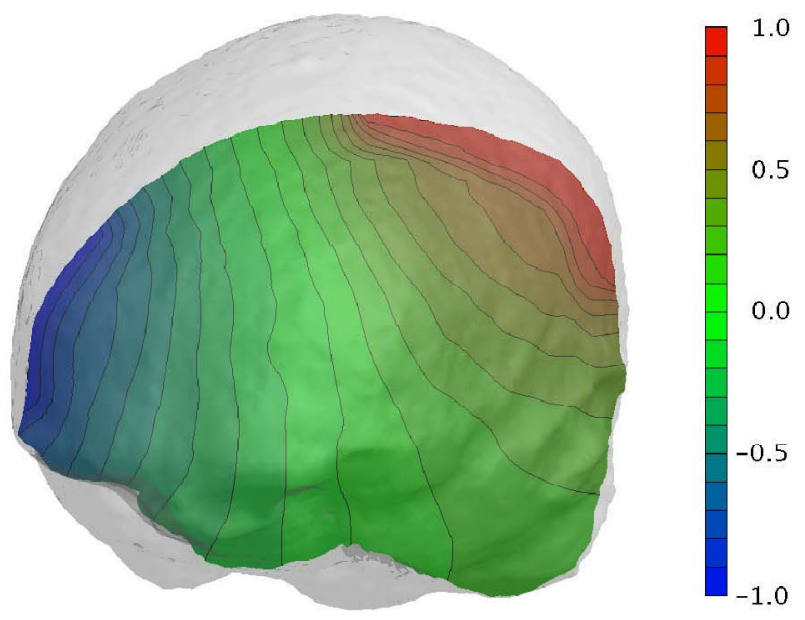

B

both the isotropic and the anisotropic model. The plane of the cross section is such that both stimulation electrodes cross it. The plot shows that the skull of the anisotropic model, as expected, is an important barrier to the current; a substantial part of the total voltage drop occurs across the skull.

Fig. 4 displays lines following the gradient of the potential from one electrode to the other. Although for anisotropic conductivities the direction of the gradient of the potential is not the same as the direction of the current, this plot gives an overall impression of the pathways of the current through the head.

The computations took about 2 minutes on a quad core Intel Xeon Mac running at $2.66 \mathrm{GHz}$ with $2 \mathrm{~GB}$ of memory.

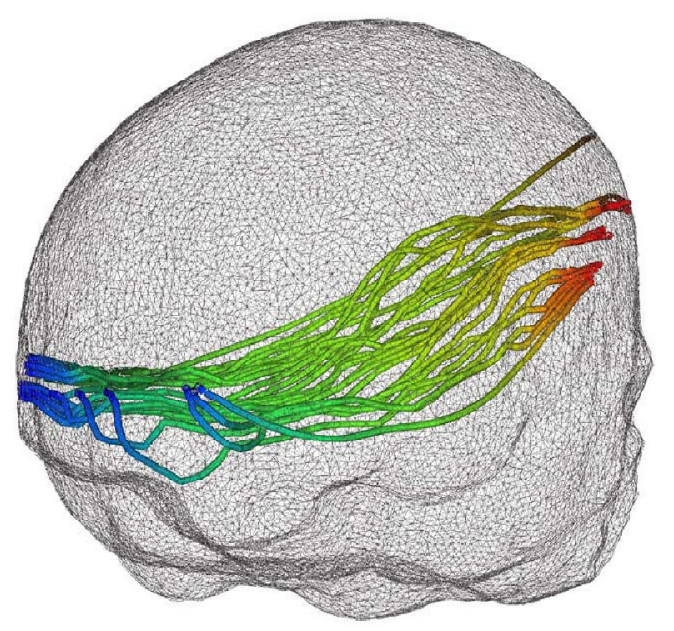

Fig. 4. The lines in this plot follow the gradient of the potential from one electrode to the other, thus giving an impression of the current lines (anisotropic model). The color of the lines corresponds to the potential at that location, using the same color scale as fig. 2 and 3. 


\section{Discussion}

The purpose of this pilot study was to estimate the potential distribution inside the human head generated by large DC stimulation electrodes on the scalp in a realistic volume conductor model that takes the anisotropy of the conductivity into account. This study shows that this is feasible. Overall, the results are qualitatively in accordance with the expectations.

There are some limitations to the model used. The modeling of the skull as a uniform, anisotropic conductor has recently been shown to be sub-optimal [16]. A model in which the skull consists of 3 isotropic layers with different conductivities, representing the layers of different bone density within the skull, seems more appropriate.

The way in which the anisotropic conductivities of the white matter were determined is sub-optimal as these tensors were modeled to be prolate (cigar-shaped), rotationally symmetric ellipsoids. While this assumption is valid in most white matter regions [17], our future work will use a model which does not rely on such assumptions since the conductivity tensor eigenvalues will be computed directly from the measured diffusion tensor eigenvalues as reported by [18].

The limitations mentioned above will be addressed in the follow-up research. The effect of the conductivity values and anisotropy ratios of the different tissues on the current distribution will be studied. The potential distribution at the scalp as computed by the FEM will be compared to the measured potentials distribution as a necessary "first order" confirmation of the model predictions. When this appears to be relevant, even individual head models can be constructed for the subjects.

The potential at the tDCS electrodes was assumed to be constant. Actually, during stimulation, a double layer is formed at the boundary between the electrode and the skin. At the electrode side of the interface the potential is truly constant, but at the skin side it will not be. Modeling this interface between the electrode and the skin will be a part of the follow-up of this work.

One subject reported visual sensations during tDCS. This might be caused by current flowing through the holes via which the optical nerves cross the skull. The current model does not include these holes. In a next version they will be included, and their effect will be studied.

As a next step, the current density distribution within the head will be computed for different electrode configurations. Finally, the correlation between this distribution and the observed behavioral effects of the DC stimulation will be evaluated. In this way, the tools developed in this study will help to understand the mechanism behind the effect of tDCS, and to plan stimulation protocols for different purposes.

\section{REFERENCES}

[1] T. Furubayashi, Y. Terao, N. Arai, S. Okabe, H. Mochizuku, R. Hanajima, M. Hamada, A. Yugeta, S. Inomata-Terada, Y. Ugawa, "Short and long duration transcranial direct current stimulation (tDCS) over the human hand motor area," Exp Brain Res, vol. 185 , pp. 279-286, 2008.

[2] M.A. Nitsche, W. Paulus, "Sustained excitability elevations induced by transcranial DC motor cortex stimulation in humans", Neurology vol. 57 pp. 1899-1901, 2001.

[3] M.A. Nitsche, M.S. Nitsche, C.C. Klein, F. Tergau, J.C. Rothwell, W. Paulus, "Level of action of cathodal DC polarisation induced inhibition of the human motor cortex," Clin Neurophysiol, vol. 114 pp. 600-604, 2003.

[4] M.A. Nitsche, K. Fricke, U. Henschke, A. Schitterlau, D. Liebetanz, N. Lang, S. Henning, F. Terfau, W. Paulus, "Pharmacoligical modulation of cortical excitability shifts induced by transcranial DC stimulation," J Physiol, vol. 553, pp. 293-301, 2003.

[5] F. Fregni, P.S. Boggio, M. Nitsche, F. Bermpohl, A. Antal, E. Feredoes, M.A. Marcolin, S.P. Rigonatti, M.T.A. Silva, W. Paulus, A. Pascual-Leone, "Anodal transeranial direct current stimulation of prefrontal cortex enhances working memory", Exp Brain Res vol. 66, pp. 23-30, 2005.

[6] M.A. Nitsche, S. Doemkes, T. Karakose, A. Antal, D. Liebetanz, N. Lang, F. Tergau, W. Paulus. "Shaping the effects of transcranial direct current stimulation of the human motor cortex", J Neurophysiol, vol. 97, pp. 109-3117, 2007.

[7] T. Wagner, F. Fregni, S. Fecteau, A. Grodzinsky, M. Zahn, A. Pascual-Leone, "Transcranial direct current stimulation: a computerbased human model study", Neurolmage, vol. 35, pp.1113-1124, 2007.

[8] C. Im, H., Jung, J. Choi, S. Y. Lee, K. Jung, "Determination of optimal electrode positions for transcranial direct current stimulation", Phys Med Biol, vol. 53, pp. N219-N225, 2008.

[9] P.C. Miranda, M. Lomarev, M. Hallet, "Modeling the current distribution during transcranial direct current stimulation", Clin Neurophysiol, vol. 117, pp. 1623-1629, 2006.

[10] S.P. van den Broek, F. Reinders, M. Donderwinkel, M.J. Peters, "Volume conduction effects in EEG and MEG", Clin Neurophys, vol. 106, pp. 522-534, 1997.

[11] C.H. Wolters, A. Anwander, X. Tricoche, D. Weinstein, M.A. Koch, R.S. MacLeod, "Influence of tissue anisotropy on EEG/MEG field and return current computation in a realistic head model: A simulation and visualization study using high resolution finite element modeling", Neurolmage, vol. 30, pp. 813-826, 2006.

[12] T.F. Oostendorp, J.Delbeke, D.F. Stegeman, "The conductivity of the human skull: Results of in vivo and in vitro measurements", IEEE Trans Biomed Eng, vol 47, pp. 1487-1491, 2000.

[13] S. Baumann, D. Wozny, S. Kelly, F. Meno, "The electrical conductivity of human cerebrospinal fluid at body temperature", IEEE Trans Biomed Eng, vol. 44, pp. 220-223, 1997.

[14] J. Haueisen, C. Ramon, M. Eiselt, H. Brauer, H. Nowak, "Influence of tissue resistivities on neuromagnetic fields and electric potentials studied with a finite element model of the head", IEEE Trans Biomed Eng, vol. 44, pp. 727-735, 1997.

[15] H. Hallez, B. Vanrumste, R. Grech, J. Muscat, W. de Clerq, A Vergult, Y. D'Asseler, K.P. Camilleri, S.G. Fabri, S. van Huffel, I. Lemahieu, "Review on solving the forward problem in EEG source analysis", J NeuroEng Rehab, vol. 4, pp. 46-75, 2007.

[16] R.J. Sadleir, A. Argibay, "Modeling Skull Electrical Properties", Ann Biomed Eng, vol. 35, pp. 1699-1712, 2007.

[17] J.S. Shimony, R.C. McKinstry, E. Akbudak, J.A. Aronovitz, A.Z. Snyder, N.F. Lori, T.S. Cull and T.E. Conturo, "Quantitative diffusion-tensor anisotropy brain MR imaging: normative human data and anatomic analysis", Radiology, vol. 212, pp.770-784, 1999.

[18] D.S. Tuch, V.J. Wedeen, A.M. Dale, J.S. George, J.W. Belliveau, "Conductivity tensor mapping of the human brain using diffusion tensor MRI", Proc Natl Acad Sci USA, vol. 98, pp. 11697-701, 2001. 